\title{
Inflammation-driven malnutrition: a new screening tool predicts outcome in Crohn's disease
}

\author{
Irene Jansen ${ }^{1}$, Matthias Prager ${ }^{1}$, Luzia Valentini ${ }^{2}$ and Carsten Büning ${ }^{1,3 *}$ \\ ${ }^{1}$ Department of Hepatology and Gastroenterology, Charité - Universitätsmedizin, Charitéplatz 1, 10117 Berlin, Germany \\ ${ }^{2}$ Unit of Dietetics, University of Applied Sciences, Brodaer Str. 2, 17033 Neubrandenburg, Germany \\ ${ }^{3}$ Innere Abteilung, Krankenhaus Waldfriede, Argentinische Allee 40, 14163 Berlin, Germany \\ (Submitted 18 May 2016 - Final revision received 14 July 2016 - Accepted 18 July 2016)
}

\begin{abstract}
Malnutrition is a frequent feature in Crohn's disease (CD), affects patient outcome and must be recognised. For chronic inflammatory diseases, recent guidelines recommend the development of combined malnutrition and inflammation risk scores. We aimed to design and evaluate a new screening tool that combines both malnutrition and inflammation parameters that might help predict clinical outcome. In a prospective cohort study, we examined fifty-five patients with CD in remission (Crohn's disease activity index (CDAI) <200) at 0 and 6 months. We assessed disease activity (CDAI, Harvey-Bradshaw index), inflammation (C-reactive protein (CRP), faecal calprotectin (FC)), malnutrition (BMI, subjective global assessment (SGA), serum albumin, handgrip strength), body composition (bioelectrical impedance analysis) and administered the newly developed 'Malnutrition Inflammation Risk Tool' (MIRT; containing BMI, unintentional weight loss over 3 months and CRP). All parameters were evaluated regarding their ability to predict disease outcome prospectively at 6 months. At baseline, more than one-third of patients showed elevated inflammatory markers despite clinical remission $(36.4 \% \mathrm{CRP} \geq 5 \mathrm{mg} / \mathrm{l}, 41.5 \% \mathrm{FC} \geq 100 \mu \mathrm{g} / \mathrm{g})$. Prevalence of malnutrition at baseline according to BMI, SGA and serum albumin was $2-16 \%$. At 6 months, MIRT significantly predicted outcome in numerous nutritional and clinical parameters (SGA, CD-related flares, hospitalisations and surgeries). In contrast, SGA, handgrip strength, BMI, albumin and body composition had no influence on the clinical course. The newly developed MIRT was found to reliably predict clinical outcome in CD patients. This screening tool might be used to facilitate clinical decision making, including treatment of both inflammation and malnutrition in order to prevent complications.
\end{abstract}

\section{Key words: Malnutrition: Inflammation: Crohn's disease: Malnutrition Inflammation Risk Tool}

Malnutrition is a frequent problem in patients with Crohn's disease (CD). Causes are multiple and include anorexia, reduced intestinal absorption, intestinal nutrient loss, drug interactions and small bowel resections. During active disease, up to $75 \%$ of patients are affected ${ }^{(1)}$, contributing to increased morbidity and mortality ${ }^{(2)}$. It affects patients' quality of life and outcome and must be treated ${ }^{(3,4)}$.

Recognition of malnutrition is not standardised, and no screening tool exists specifically for inflammatory bowel diseases (IBD). Furthermore, in chronic inflammatory diseases such as $\mathrm{CD}$, inflammation plays a central role in the pathophysiology of disease-related malnutrition. Inflammation in malnutrition limits the effectiveness of nutritional therapy and requires treatment of the underlying disease. None of the tools recommended by the European Society for Clinical Nutrition and Metabolism (ESPEN) and the American Society for Parenteral and Enteral Nutrition (ASPEN), such as the Nutritional Risk Screening (NRS-2002), Malnutrition Universal Screening Tool (MUST) or the subjective global assessment
(SGA), contains inflammatory markers, although both ESPEN and ASPEN recently postulated an aetiology-based definition of malnutrition and define 'chronic disease-related malnutrition' as a separate category ${ }^{(5,6)}$. ESPEN recommends MUST in the ambulatory care setting. However, evidence is lacking that MUST has influence on clinical outcome in this group of patients. The aim of the present study was to develop an easy-to-use screening tool for the ambulatory care setting that combines malnutrition and inflammation parameters. We evaluated its potential to predict disease outcome in patients with a chronic inflammatory condition.

\section{Methods}

\section{Study participants}

Between September 2012 and July 2013, we included fifty-five patients diagnosed with CD according to standard criteria. Participants were recruited from the outpatient IBD clinic at the Department of Gastroenterology and Hepatology of the Charité,

Abbreviations: CD, Crohn's disease; CDAI, Crohn's disease activity index; CRP, C-reactive protein; FC, faecal calprotectin; HBI, Harvey-Bradshaw index; MIRT, Malnutrition Inflammation Risk Tool; MUST, Malnutrition Universal Screening Tool; NRS, Nutritional Risk Screening; SGA, subjective global assessment.

* Corresponding author: C. Büning, fax +49 30 8181077243, email c.buening@waldfriede.de 
Universitätsmedizin, Berlin. Inclusion criteria were Crohn's disease activity index (CDAI) $<200$, age $18-75$ years, occurrence of relapse/flare-ups, intestinal complication or hospitalisation within the last 2 years, endoscopic diagnosis to identify disease location and behaviour within the last 2 years. Exclusion criteria were presence of cancer or other severe disease, pregnancy or lactation, high-dose systemic corticosteroid treatment within 3 months before study entry, presence of stoma or short bowel syndrome and BMI $<17.5$ or $<20 \mathrm{~kg} / \mathrm{m}^{2}$ plus severe weight loss ( $>10 \%$ within previous 6 months before study entry), as these conditions would have implicated direct nutritional treatment.

\section{Study protocol}

In a prospective cohort study, we examined the patients twice: at baseline and after 6 months. The following data of $\mathrm{CD}$ patients were obtained: sex, age, disease localisation and behaviour according to the Montreal classification ${ }^{(7)}$ and drug treatment at baseline. During the study period, they were asked to fill out a detailed protocol regarding any kind of diseaserelated complications and changes in CD medication. The following investigations were performed on both study days (for detailed description see below): calculation of disease activity, blood and stool analysis, and assessment of nutritional status and body composition. We, furthermore, collected data on CDrelated events (doctor visits, complications such as stenosis, fistula or abscess, hospitalisations, flares, surgery and changes in medications; for definitions see Table 1). In addition, each event was scored (points ranging from -1 to +5 ) and integrated into a composite assessment (Table 1).

\section{Clinical disease activity}

To assess disease activity, we used the $\mathrm{CDAI}^{(8)}$ as well as the Harvey-Bradshaw index $(\mathrm{HBI})^{(9)}$. The CDAI is the established gold standard for assessing disease activity. Main components of the CDAI are patient-reported symptoms such as diarrhoea, abdominal pain and overall well-being referring to the last week before assessment. Furthermore, it includes, for example, the presence of extra-intestinal manifestations, haematocrit and ratio of actual body weight:standard body weight. The HBI, which has shown good correlation with the CDAI, is a shorter version and simpler to use ${ }^{(10)}$.

\section{Laboratory parameters}

Venous blood samples were obtained from all patients after an overnight fast. Albumin, C-reactive protein (CRP) and blood count (leucocytes, hb) were analysed at the routine laboratory. For analysis of faecal calprotectin (FC), forty-one patients at baseline and thirty-seven patients after 6 months provided faecal samples on the day or the day before the examination. The samples were frozen at $-20^{\circ} \mathrm{C}$ until analysis in the laboratory for endocrinology of the Charité, Universitätsmedizin, Berlin. FC was analysed in duplicate using the kit $\mathrm{PhiCal}^{\circledR}$ Calprotectin-ELISAKit (MRP 8/14, stool; Immundiagnostik AG). The reference values given by the manufacturer were $<50 \mu \mathrm{g} / \mathrm{g}$. Assays were applied according to the manufacturers' instructions.

\section{Malnutrition}

Subjective global assessment ${ }^{(11)}$. The SGA combines anamnestic data with a physical examination. Medical history included weight and weight changes, nutritional intake, gastrointestinal symptoms and state of disease. The physical examination focused on loss of subcutaneous fat, muscle atrophy, oedema and ascites. The patient was then classified into category A (well nourished), B (mild-to-moderately malnourished) or C (severely malnourished).

Anthropometry. Height and weight were assessed using the digital stadiometer 274 (Seca), and BMI was calculated as body weight $(\mathrm{kg}) /$ body height $\left(\mathrm{m}^{2}\right)$.

Bioelectrical impedance analysis. We used the medical Body Composition Analyzer (mBCA) seca 515 (Seca) and the corresponding software seca analytics mBCA 115. Measurements were taken in subjects after an 8-h overnight fast, voiding of the urinary bladder and standing upright for at least $10 \mathrm{~min}$.

Table 1. Clinical parameters collected prospectively (summarised in a composite assessment)

\begin{tabular}{lll}
\hline Criteria & Explanation & Points \\
\hline CD-related doctor visits & IBD clinic, general practitioner, emergency department & $+1 /$ visit \\
Complications & Newly occurred stenosis, fistula or abscess & $+3 /$ complication \\
CD-associated hospitalisation & & $+1 / \mathrm{d}$ \\
Flare-up/relapse & & $+5 /$ relapse \\
CD-related surgery & $\uparrow$ Prednisolone & $+5 /$ surgery \\
Changes in CD medication & $\uparrow$ Anti-TNF agents & +1 \\
& $\uparrow$ Immunosuppressants & +1 \\
& $\downarrow$ Prednisolone & +1 \\
& $\downarrow$ Anti-TNF agents & -1 \\
& $\downarrow$ Immunosuppressants & -1 \\
& Intake of antibiotics $>2$ d & -1
\end{tabular}

Sum

CD, Crohn's disease; IBD, inflammatory bowel diseases; NSAID, non-steroidal anti-inflammatory drug (e.g. aspirin, diclofenac and ibuprofen; not 5 -aminosalicylic acid/mesalazine); assessment: values $\geq 5$ points were evaluated as a worsening course of disease. 
Table 2. Malnutrition Inflammation Risk Tool*

\begin{tabular}{lrrrrrr}
\hline & & Points & & Points & & Points \\
\hline BMl $\left(\mathrm{kg} / \mathrm{m}^{2}\right)$ & $>20$ & 0 & $18 \cdot 5-20 \cdot 0$ & 1 & $<18.5$ & 2 \\
Weight loss & $<5$ & 0 & $5-<10$ & 2 & $\geq 10$ & 3 \\
$\quad 3$ months $(\%)$ & & & & & & \\
CRP (mg/l) & $<5$ & 0 & $5-50$ & 2 & $\geq 50$ & 3 \\
\hline
\end{tabular}

* Score: 0-8 points.

Handgrip strength. Handgrip strength was recorded using the Jamar vigorimeter (Preston). Participants were ask to sit with the shoulder adducted, the elbow positioned on a table and flexed to $90^{\circ}$. A maximal contraction of the dominant hand was performed. This was repeated three times with a 45 -s break in between, and the highest value was recorded.

Malnutrition Inflammation Risk Tool. On the basis of nutrition-associated data of approximately $100 \mathrm{CD}$ patients of previous studies ${ }^{(12,13)}$, we developed the Malnutrition Inflammation Risk Tool (MIRT) (Table 2).

\section{Statistical considerations}

Data are expressed as mean values and standard deviations if not indicated otherwise. The Kolmogorov-Smirnov test was used to evaluate the normal distribution of data sets. As there was no normal distribution, we assessed differences between groups by non-parametric Mann-Whitney $U$ test and for more than two groups the Kruskal-Wallis test. Linear correlations were calculated using Spearman's rank-order correlation coefficient. Results were considered significant if $P$ values were $<0 \cdot 05$. All analyses were performed using SPSS, version 22.0 (SPSS Inc., IBM).

\section{Ethics statement}

The study protocol was approved by the ethics committee of the Charité, Universitätsmedizin, Berlin (ref. EA4/059/13). Informed consent was obtained from all study participants before study entry.

\section{Results}

\section{Characteristics of Crohn's disease patients}

Demographic and clinical characteristics of the sample are presented in Table 3 and partly in Table 4.

\section{Inflammation and malnutrition parameters at baseline}

BMI categories defined by the WHO showed $2 \%$ underweight, $56 \%$ normal weight, $29 \%$ overweight and $13 \%$ obesity. In total, $2-16 \%$ of CD patients showed signs of malnutrition according to BMI, albumin, SGA and 'unintended weight loss' (Table 4). Disease duration did not correlate with SGA. In all, twenty patients (36.4\%) had a CRP level $>5 \mathrm{mg} / \mathrm{l}$. Among them, there were two ( $4.0 \%$ ) who showed values $>50 \mathrm{mg} / \mathrm{l}$. In total,
Table 3. Baseline characteristics of Crohn's disease patients (Absolute numbers and percentages; mean values and standard deviations; $n$ 55)

\begin{tabular}{|c|c|c|c|}
\hline & $n$ & & $\%$ \\
\hline Female & 36 & & 65.4 \\
\hline \multicolumn{4}{|l|}{ Age (years) } \\
\hline Mean & & 40 & \\
\hline SD & & 11 & \\
\hline \multicolumn{4}{|l|}{ Disease duration (years) } \\
\hline Mean & & 9 & \\
\hline SD & & $7 \cdot 7$ & \\
\hline \multicolumn{4}{|l|}{ CDAI } \\
\hline Mean & & 83 & \\
\hline SD & & 52 & \\
\hline \multicolumn{4}{|l|}{$\mathrm{HBI}$} \\
\hline Mean & & 4 & \\
\hline SD & & 3 & \\
\hline \multicolumn{4}{|l|}{$\mathrm{Hb}(\mathrm{g} / \mathrm{l})$} \\
\hline Mean & & 135 & \\
\hline SD & & 16 & \\
\hline \multicolumn{4}{|l|}{ Leucocytes (g/l) } \\
\hline Mean & & $17 \cdot 6$ & \\
\hline SD & & $2 \cdot 6$ & \\
\hline \multicolumn{4}{|l|}{ Montreal classification ${ }^{(7)}$} \\
\hline \multicolumn{4}{|l|}{ Age } \\
\hline $\mathrm{A} 1$ & 2 & & 3.6 \\
\hline $\mathrm{A} 2$ & 41 & & 74.5 \\
\hline A3 & 12 & & $21 \cdot 8$ \\
\hline \multicolumn{4}{|l|}{ Localisation } \\
\hline L1 & 10 & & $18 \cdot 1$ \\
\hline L2 & 20 & & $36 \cdot 4$ \\
\hline L3 & 16 & & $29 \cdot 1$ \\
\hline L4/L4+ & $2 / 7$ & & $3 \cdot 6 / 12 \cdot 7$ \\
\hline \multicolumn{4}{|l|}{ Behaviour } \\
\hline B1 & 27 & & $49 \cdot 1$ \\
\hline B2 & 13 & & 23.6 \\
\hline B3 & 15 & & $27 \cdot 2$ \\
\hline B3p & 20 & & $36 \cdot 4$ \\
\hline \multicolumn{4}{|l|}{ Drug treatment } \\
\hline No medication & 5 & & $9 \cdot 1$ \\
\hline \multicolumn{4}{|l|}{ Glucocorticoids } \\
\hline Prednisolone $(<20 \mathrm{mg} / \mathrm{d})$ & 10 & & $18 \cdot 2$ \\
\hline Oral budesonide & 3 & & 5.5 \\
\hline Azathioprine & 29 & & $52 \cdot 7$ \\
\hline Mesalazine & 4 & & $7 \cdot 3$ \\
\hline Methotrexate & 2 & & 3.6 \\
\hline \multicolumn{4}{|l|}{ Anti TNF- $a$} \\
\hline Infliximab & 15 & & $27 \cdot 3$ \\
\hline Adalimumab & 6 & & 10.9 \\
\hline
\end{tabular}

CDAI, Crohn's disease activity index; HBI, Harvey-Bradshaw index.

seventeen patients $(41.5 \%, n$ 41) had FC levels $\geq 100 \mu \mathrm{g} / \mathrm{g}$. CRP and FC correlated significantly ( $r 0.467, P=0 \cdot 002$, Spearman). In all, nine patients had albumin levels $<395 \mathrm{mg} / \mathrm{l}$. Among these, there was one patient $(2 \cdot 7 \%)$ whose level was $<360 \mathrm{mg} / \mathrm{l}$. There was an inverse correlation both between CRP and albumin $(r-0.344, P=0 \cdot 010$, Spearman $)$ and FC and albumin $(r-0 \cdot 505, P=0 \cdot 001$, Spearman). In total, nine (16.4\%) patients showed a MIRT $\geq 3$.

\section{Clinical course of disease after 6 months}

The clinical outcome was measured by disease activity (CDAI, HBI), malnutrition (SGA) and the following clinical parameters that occurred during the 6-month study period: days spent in 
Table 4. Clinical, inflammatory and nutritional characteristics of Crohn's disease patients

(Mean values and standard deviations; absolute numbers and percentages)

\begin{tabular}{|c|c|c|c|c|}
\hline & \multicolumn{2}{|c|}{ Baseline } & \multicolumn{2}{|c|}{ After 6 months } \\
\hline & Mean & SD & Mean & SD \\
\hline \multicolumn{5}{|l|}{ Disease activity } \\
\hline CDAI & 83 & 52 & 79 & 62 \\
\hline $\mathrm{HBI}$ & 4 & 3 & 4 & 3 \\
\hline \multicolumn{5}{|l|}{ Inflammation } \\
\hline CRP (mg/l) & $8 \cdot 1$ & $15 \cdot 1$ & $5 \cdot 1$ & 8.4 \\
\hline $\mathrm{FC}(\mu \mathrm{g} / \mathrm{g})$ & $168 \cdot 7$ & $230 \cdot 6$ & $297 \cdot 2$ & $363 \cdot 2$ \\
\hline \multicolumn{5}{|l|}{ Nutrition } \\
\hline BMI $\left(\mathrm{kg} / \mathrm{m}^{2}\right)$ & 24.9 & $5 \cdot 0$ & $25 \cdot 6$ & 4.9 \\
\hline Handgrip strength $(\mathrm{kg})$ & $38 \cdot 2$ & $9 \cdot 9$ & $37 \cdot 9$ & $10 \cdot 0$ \\
\hline Phase angle $\left({ }^{\circ}\right)$ & $5 \cdot 0$ & 0.6 & $5 \cdot 1$ & 0.6 \\
\hline Fat mass $(\mathrm{kg})$ & $23 \cdot 2$ & $10 \cdot 5$ & $24 \cdot 4$ & $10 \cdot 8$ \\
\hline Body cell mass $(\mathrm{kg})$ & 23.9 & $6 \cdot 0$ & $24 \cdot 3$ & $6 \cdot 0$ \\
\hline \multicolumn{5}{|l|}{ SGA B or $C$} \\
\hline$n$ & \multicolumn{2}{|c|}{7} & \multicolumn{2}{|c|}{3} \\
\hline$\%$ & \multicolumn{2}{|c|}{$12 \cdot 7$} & \multicolumn{2}{|c|}{$6 \cdot 0$} \\
\hline Albumin (mg/l) & 436 & 37 & 445 & 36 \\
\hline MIRT & 1 & 1 & & \\
\hline \multicolumn{5}{|l|}{ Clinical parameters } \\
\hline Days in hospital & \multicolumn{2}{|c|}{-} & 1.8 & $5 \cdot 2$ \\
\hline Number of flare-ups & \multicolumn{2}{|c|}{-} & 0.3 & 0.8 \\
\hline Number of complications* & \multicolumn{2}{|c|}{-} & 0.7 & 1.7 \\
\hline Number of surgeries & \multirow{2}{*}{\multicolumn{2}{|c|}{-}} & 0.1 & 0.4 \\
\hline Composite assessment & & & 8 & 14 \\
\hline
\end{tabular}

CDAI, Crohn's disease activity index; HBI, Harvey-Bradshaw index; CRP, C-reactive protein; FC, faecal calprotectin; SGA, subjective global assessment; MIRT, Malnutrition Inflammation Risk Tool.

* Occurrence of stenosis, fistula or abscess within the 6-month study period.

hospital, number of flare-ups diagnosed by a senior physician, number of complications (new stenosis, fistula or abscess), number of CD-related surgeries and changes in drug treatment (Table 4). In addition, these parameters were summarised in a composite assessment (see Table 1). Among all, five patients did not attend assessment after 6 months. It was still possible to collect data on CD-related events in three patients via telephone interviews and electronic data; thus, data on fifty-three patients (96.4\%) were available at 6 months. In all, eleven patients (20.8\%) were diagnosed with at least one flare-up within the study period. In addition, twelve patients (22.6\%) suffered from complications (stenosis, fistula, abscess), eleven (20.8\%) were admitted to the hospital and six (11.3\%) had to undergo surgery. More than one-third (39.6\%) of patients suffered from one of these four clinical conditions. Composite assessment ranged from 0 to 67 , and the mean value was 8 (sD 14).

\section{Malnutrition Inflammation Risk Tool and outcome at 6 months}

Spearman's correlation analysis was performed between MIRT and numerous parameters (Table 5). In this case, MIRT predicted outcome for SGA and clinical parameters such as hospitalisations, surgeries, complications and flares. MIRT values $\geq 3$ showed an relative risk of $4 \cdot 0(2 \cdot 4-6 \cdot 7)$ for worsening of clinical outcomes (clinical parameters are summarised in the composite assessment). The two components of MIRT - 'weight loss' and
Table 5. Influence of Malnutrition Inflammation Risk Tool (MIRT) on outcome parameters after 6 months

\begin{tabular}{lcr}
\hline & \multicolumn{2}{c}{ MIRT } \\
\cline { 2 - 3 } & $R^{\star}$ & $P$ \\
\hline Disease activity & & \\
CDAI & 0.260 & 0.077 \\
HBI & 0.188 & 0.195 \\
Nutrition & & \\
SGA & 0.394 & 0.005 \\
Clinical parameters & & \\
$\quad$ Hospitalisations & 0.398 & 0.003 \\
$\quad$ Flares & 0.299 & 0.030 \\
Complications & 0.333 & 0.015 \\
$\quad$ Surgeries & 0.371 & 0.006 \\
Composite assessment & 0.528 & $<0.001$ \\
\hline
\end{tabular}

CDAI, Crohn's disease activity index; HBI, Harvey-Bradshaw index; SGA, subjective global assessment.

* Spearman's correlation.

CRP - correlated individually with various outcomes (weight loss: complications $P=0.003$, surgeries $P=0.020$, composite assessment $P=0.013$; CRP: SGA $P=0.034$, hospitalisation $P=0 \cdot 008$, surgeries $P=0 \cdot 010$, composite assessment $P=0 \cdot 008$ ). FC was also associated with clinical outcome (complications $P=0 \cdot 011$, surgeries $P=0 \cdot 022$, composite assessment $P=0 \cdot 008$ ). All other parameters such as SGA, albumin, BMI, body composition (phase angle) and handgrip strength had no significant influence on all investigated outcome parameters.

\section{Discussion}

In this prospective cohort study including fifty-five patients with $\mathrm{CD}$ in remission, we showed that the newly developed MIRT predicts clinical outcome after 6 months. CRP, FC and unintended weight loss proved to be independent predictive factors, whereas BMI, handgrip strength, bioelectrical impedance analysis (BIA) and SGA did not correlate with the clinical outcome.

\section{Course of disease}

Evaluation of the clinical course in $\mathrm{CD}$ is a challenge, given the complex and varying character of the disease with numerous relevant parameters to consider. We measured established CD- and nutrition-related end points - for example, CDAI, HBI and SGA. However, CDAI and HBI are criticised for their low specificity due to their emphasis on subjective parameters ${ }^{(14)}$. We, furthermore, collected data on CD-related doctor visits, disease flare-ups, hospitalisations, surgeries and changes in CD medication. For better overview, these data are summarised in a composite assessment. In this case, approximately one-third (37.7\%) of our study patients ( $\geq 5$ points in the composite assessment) developed worsening of $\mathrm{CD}$, which is in line with data of population-based studies investigating the clinical course of $\mathrm{CD}^{(15)}$.

\section{Malnutrition}

Data regarding nutritional status of CD patients in remission are scarce and results are conflicting, which might be due to the 
lack of a standard assessment tool ${ }^{(12)}$. Almost half of our patients were either overweight or obese. This high percentage is congruent with recent epidemiological data on CD patients in Germany ${ }^{(16)}$. Consequently, BMI alone should not be used as a tool for detecting malnutrition, as obese patients will never reach the threshold of $<18.5 \mathrm{~kg} / \mathrm{m}^{2}$ even in extreme catabolism. The prevalence of malnutrition at baseline and after 6 months was $2-16 \%$, which is in accordance with previous studies examining nutritional status of CD patients in remission ${ }^{(12,17,18)}$. BIA at both study days showed impaired body composition characterised by higher fat mass and lower body cell mass compared with previous studies ${ }^{(12)}$. This could reflect a chronic inflammatory condition. Surprisingly, handgrip strength was higher at both times in the current study group compared with an earlier study from our institution ${ }^{(12)}$. Handgrip strength is a simple measurement of muscle function that has high sensitivity in detecting disease-related malnutrition. It is a good predictor of clinical outcome measured by postoperative complications, length of hospitalisation, decreased physical activity status and mortality in various chronic diseases, but not in $\mathrm{CD}^{(19)}$. However, in our study, handgrip strength did not correlate with disease course and predictive value could not be confirmed. BMI values did not show good linear correlations to clinical outcome, but when BMI groups were compared there was a tendency towards a less-favourable course for both patients who were underweight and patients who were obese (data not shown). Several studies suggest that obesity pre-destines for a more aggressive course, as obese patients are found to suffer more frequently from flare-ups, perianal fistulas and hospitalisation $^{(20)}$. The nutritional parameter with the highest associations with clinical outcome in our study was "unintentional weight loss'. Its relevance is reflected by its use in many nutritional tools (SGA, NRS-2002, Mini Nutritional Assessment, MUST) as it is considered the best indicator for malnutrition. Its negative influence on surgical complications in $\mathrm{CD}$ has been described earlier ${ }^{(21)}$. SGA, serum albumin and phase angle did not show relevant associations with clinical course. In contrast to this, phase angle was a reliable predictor of clinical outcome in a recent study ${ }^{(22)}$. However, this study was performed in oncology patients, and therefore comparability with our data is limited. Investigations regarding the predictive value of SGA and albumin have shown higher risk of mortality, but none of the studies were performed in patients with $\operatorname{IBD}^{(23,24)}$. All these observations show that current tools are insufficient to predict the clinical course of $\mathrm{CD}$.

\section{Inflammation}

Despite clinical remission, raised inflammatory markers were present at baseline assessment in more than a third of patients. This proportion is in line with other results regarding persisting subclinical inflammation in $\mathrm{CD}^{(25)}$. The CRP proved to be a strong individual predictor of clinical outcome in our study. It correlated with SGA, days in hospital, number of surgeries and with the composite assessment. Its predictive potential in CD patients has been investigated before. In a population-based study, patients with elevated CRP had a higher risk for surgeries $^{(25)}$. On the other hand, normal CRP levels were shown to predict better response to nutritional therapy and closing of enterocutaneous fistulas ${ }^{(26)}$. The main disadvantage of CRP is its low specificity as an acute-phase protein. This is why research on faecal inflammatory markers such as calprotectin and lactoferrin has increased. FC highly correlates with endoscopically identified mucosal inflammation of the gastrointestinal tract ${ }^{(27)}$. It is highly used in clinical practice to diagnose, predict relapse and control therapy ${ }^{(28,29)}$. In our study, it was found to be an independent individual predictor of clinical outcome, although the correlations were not as strong as CRP. This might be due to the smaller sample size of only forty-one patients who delivered a faecal sample at baseline assessment. As we could not detect superiority of FC over CRP, FC does not need to be integrated in the MIRT, especially because stool collection remains a challenge as shown in our study. However, our data underline the importance of regularly measuring inflammatory markers to monitor clinical course of $\mathrm{CD}$.

\section{Malnutrition Inflammation Risk Tool}

The MIRT was developed as a simple tool composed of the objective parameters BMI, unintended weight loss and CRP in order to combine both nutrition- and inflammation-related measures. In our study, the tool proved to be highly predictive of clinical outcome. It showed significant associations with the following individual clinical end points: CD-related days in hospital, number of flares, complications (newly occurred stenosis, fistula or abscess) and CD-related surgeries. Furthermore, it correlated with the validated nutritional outcome SGA. Finally, it showed a tendency towards an association with the CDAI. Nevertheless, the relationship failed statistical significance, which might be due to previously discussed limitations of these activity scores.

\section{Malnutrition Inflammation Risk Tool in comparison with standard malnutrition screening tools}

The two standard tools MUST and NRS-2002 do not contain an objective inflammatory parameter such as CRP. MUST contains BMI, unexplained weight loss and acute illness with no nutritional intake $>5 \mathrm{~d}$. NRS-2002 includes within the pre-screening BMI, unexplained weight loss, reduced energy intake and also ongoing illness. MIRT contains BMI and unintended weight loss but also includes an inflammatory marker (CRP). In CD patients in remission, CRP might detect a subclinical inflammation that is relevant for disease outcome as shown in our study and others recently $^{(30)}$. We therefore believe that including CRP to the MIRT is a substantial prognostic advantage and might be in favour of MIRT. On the other hand, MUST and NRS-2002 have the important advantage in that no blood samples are required. However, in CD, CRP is a standard tool used by numerous clinicians, for example, for determining subclinical inflammation or tailoring anti-inflammatory therapy, especially with biologics. Of course, we cannot exclude the fact that both MUST and NRS-2002 have similar potential to predict disease outcome, but this was not the aim of our study. Further studies should compare the MIRT with MUST and NRS-2002 in ambulatory and inpatient $\mathrm{CD}$ patients. Of further interest would be to 
compare all three tools in other chronic diseases with high risk of malnutrition where CRP has also shown to correlate with clinical outcomes, such as solid tumours ${ }^{(31,32)}$, liver cirrhosis ${ }^{(33)}$ and even Parkinson's disease ${ }^{(34)}$.

\section{Limitations of the study}

The main limitation of our study is the restricted comparability of the MIRT due to our study design as the first evaluation of a new tool. The sample size of fifty-five patients is small but comparable with previous studies investigating nutritional status in CD patients ${ }^{(12,17,18)}$. Unfortunately, additional evaluation of inflammation with endoscopic and histological activity measured at baseline and 6 months was not performed because of ethical considerations. We therefore used FC that has shown good correlation with endoscopic activity ${ }^{(27)}$. CD is a complex disease and no standard tool exists that combines all relevant parameters to measure the course of the disease. We analysed individual CD-related events such as flares, surgeries and hospitalisations and summarised these data in a composite assessment. The evaluation of this assessment was not intended in the current protocol, but we tried to obtain a more realistic CD-related outcome with integration of relevant complications than using only standard tools such as the CDAI and HBI. Because of the observational character of our study, the ethics committee did not allow us to include patients with either active disease (CDAI >200) or more severe malnutrition disease (BMI $<17.5$ or $<20 \mathrm{~kg} / \mathrm{m}^{2}$ with severe weight loss of $>10 \% \times 6$ months), as these patients need to undergo treatment immediately. However, more studies evaluating the MIRT in active CD and malnourished patients compared with MUST and NRS-2002 should be undertaken. As we excluded patients who were severely malnourished, we cannot exclude that other nutritional parameters/scores such as the SGA might indeed correlate with clinical outcomes if these patients were analysed.

\section{Conclusions}

Both malnutrition and inflammation affect disease course in CD. MIRT was developed as an easy-to-use screening tool that combines both malnutrition and inflammation parameters. MIRT predicted clinical outcome in $\mathrm{CD}$ patients at 6 months. Clinicians could use MIRT on a regular basis to identify patients at risk and adapt medical therapy to prevent patients from complications. We hope that MIRT will be evaluated prospectively in future studies.

\section{Acknowledgements}

The authors thank Mrs Martina Werich for technical assistance.

This research received no specific grant from any funding agency or from commercial or not-for-profit sectors.

All four authors formulated the research question and designed the study. Acquisition of data was carried out by I. J., M. P. and C. B. Analysis and interpretation of data were performed by I. J., L. V. and C. B. I. J., L. V. and C. B. drafted the article, M. P. revised for important intellectual content. All authors approved the final version before submission.

The authors declare that there are no conflicts of interest.

\section{References}

1. Seidman EG (1989) Nutritional management of inflammatory bowel disease. Gastroenterol Clin North Am 18, 129-155.

2. Norman K, Pichard C, Lochs H, et al. (2008) Prognostic impact of disease-related malnutrition. Clin Nutr 27, 5-15.

3. Lochs H, Dejong C, Hammarqvist F, et al. (2006) ESPEN Guidelines on Enteral Nutrition: Gastroenterology. Clin Nutr 25, 260-274.

4. Dignass A, Van Assche G, Lindsay JO, et al. (2010) The second European evidence-based Consensus on the diagnosis and management of Crohn's disease: current management. J Crobns Colitis 4, 28-62.

5. Jensen GL, Mirtallo J, Compher C, et al. (2010) Adult starvation and disease-related malnutrition: a proposal for etiologybased diagnosis in the clinical practice setting from the International Consensus Guideline Committee. Clin Nutr 29, 151-153.

6. White JV, Guenter P, Jensen G, et al. (2012) Consensus statement: Academy of Nutrition and Dietetics and American Society for Parenteral and Enteral Nutrition: characteristics recommended for the identification and documentation of adult malnutrition (undernutrition). J Parenter Enteral Nutr 36, 275-283.

7. Silverberg MS, Satsangi J, Ahmad T, et al. (2005) Toward an integrated clinical, molecular and serological classification of inflammatory bowel disease: report of a Working Party of the 2005 Montreal World Congress of Gastroenterology. Can J Gastroenterol 19, Suppl. A, 5A-36A.

8. Best WR, Becktel JM, Singleton JW, et al. (1976) Development of a Crohn's disease activity index. National Cooperative Crohn's Disease Study. Gastroenterology 70, 439-444.

9. Harvey RF \& Bradshaw JM (1980) A simple index of Crohn's-disease activity. Lancet 1, 514.

10. Best WR (2006) Predicting the Crohn's disease activity index from the Harvey-Bradshaw Index. Inflamm Bowel Dis 12, 304-310.

11. Detsky AS, McLaughlin JR, Baker JP, et al. (1987) What is subjective global assessment of nutritional status? J Parenter Enteral Nutr 11, 8-13.

12. Valentini L, Schaper L, Buning C, et al. (2008) Malnutrition and impaired muscle strength in patients with Crohn's disease and ulcerative colitis in remission. Nutrition 24, 694-702.

13. Hengstermann S, Valentini L, Schaper L, et al. (2008) Altered status of antioxidant vitamins and fatty acids in patients with inactive inflammatory bowel disease. Clin Nutr 27, 571-578.

14. Peyrin-Biroulet L, Reinisch W, Colombel JF, et al. (2014) Clinical disease activity, C-reactive protein normalisation and mucosal healing in Crohn's disease in the SONIC trial. Gut $\mathbf{6 3}$, $88-95$.

15. Baumgart DC \& Sandborn WJ (2012) Crohn's disease. Lancet 380, 1590-1605.

16. Bokemeyer B, Hardt J, Huppe D, et al. (2013) Clinical status, psychosocial impairments, medical treatment and health care costs for patients with inflammatory bowel disease (IBD) in Germany: an online IBD registry. J Crohns Colitis 7, 355-368.

17. Bin CM, Flores C, Alvares-da-Silva MR, et al. (2010) Comparison between handgrip strength, subjective global assessment, anthropometry, and biochemical markers in assessing nutritional status of patients with Crohn's disease in clinical remission. Dig Dis Sci 55, 137-144.

18. Sousa Guerreiro C, Cravo M, Costa AR, et al. (2007) A comprehensive approach to evaluate nutritional status in Crohn's patients in the era of biologic therapy: a case-control study. Am J Gastroenterol 102, 2551-2556. 
19. Norman K, Stobaus N, Gonzalez MC, et al. (2011) Hand grip strength: outcome predictor and marker of nutritional status. Clin Nutr 30, 135-142.

20. Blain A, Cattan S, Beaugerie L, et al. (2002) Crohn's disease clinical course and severity in obese patients. Clin Nutr 21, 51-57.

21. Skowronska-Piekarska U, Matysiak K \& Sowinska A (2013) The impact of the nutritional state of patients on the results of the surgical treatment of ulcerative colitis. Pol Przegl Chir 85, 424-432.

22. Norman K, Stobaus N, Zocher D, et al. (2010) Cutoff percentiles of bioelectrical phase angle predict functionality, quality of life, and mortality in patients with cancer. Am J Clin Nutr 92, 612-619.

23. Bector S, Vagianos K, Suh M, et al. (2016) Does the Subjective Global Assessment predict outcome in critically ill medical patients? J Intensive Care Med 31, 485-489.

24. Holst M, Yifter-Lindgren E, Surowiak M, et al. (2013) Nutritional screening and risk factors in elderly hospitalized patients: association to clinical outcome? Scand J Caring Sci 27, 953-961.

25. Henriksen M, Jahnsen J, Lygren IS, et al. (2008) C-reactive protein: a predictive factor and marker of inflammation in inflammatory bowel disease. Results from a prospective population-based study. Gut 57, 1518-1523.

26. Yan D, Ren J, Wang G, et al. (2014) Predictors of response to enteral nutrition in abdominal enterocutaneous fistula patients with Crohn's disease. Eur J Clin Nutr 68, 959-963.

27. Mosli MH, Zou G, Garg SK, et al. (2015) C-reactive protein, fecal calprotectin, and stool lactoferrin for detection of endoscopic activity in symptomatic inflammatory bowel disease patients: a systematic review and meta-analysis. Am J Gastroenterol 110, 802-819.

28. Sands BE (2015) Biomarkers of inflammation in inflammatory bowel disease. Gastroenterology 149, 1275-1285.

29. Ferreiro-Iglesias R, Barreiro-de Acosta M, Otero Santiago M, et al. (2016) Fecal calprotectin as predictor of relapse in patients with inflammatory bowel disease under maintenance infliximab therapy. J Clin Gastroenterol 50, $147-151$.

30. Click B, Vargas EJ, Anderson AM, et al. (2015) Silent Crohn's disease: asymptomatic patients with elevated C-reactive protein are at risk for subsequent hospitalization. Inflamm Bowel Dis 21, 2254-2261.

31. Rekik S, Guyot E, Bhais M, et al. (2016) The CRP level and STATE score predict survival in cirrhotic patients with hepatocellular carcinoma treated by transarterial embolization. Dig Liver Dis (Epublication ahead of print version 15 June 2016).

32. Shibutani M, Maeda K, Nagahara H, et al. (2016) Prognostic significance of the preoperative ratio of C-reactive protein to albumin in patients with colorectal cancer. Anticancer Res 36, 995-1001.

33. Cervoni JP, Thévenot T, Weil D, et al. (2012) C-reactive protein predicts short-term mortality in patients with cirrhosis. J Hepatol 56, 1299-1304.

34. Sawada H, Oeda T, Umemura A, et al. (2015) Baseline C-reactive protein levels and life prognosis in Parkinson disease. PLOS ONE 10, e0134118. 